


\title{
Ecuaciones econométricas para los costos de inversión en plantas de tratamiento de agua potable en Colombia ${ }^{1}$. Econometric equation for investment costs of treatment plants in Colombia drinking water
}

\author{
León Peña Karen Liliana ${ }^{2}$, Rey Ardila Gina Lorena ${ }^{3}$, Rodríguez Miranda Juan Pablo ${ }^{4}$ \\ Universidad Distrital Francisco José de Caldas.
}

Artículo recibido en marzo de 2016; artículo aceptado en mayo de 2016

Citación del artículo: León. L, Rey L \&, Rodríguez. J. (2016). Ecuaciones Econométricas Para Los Costos De Inversión En Plantas De Tratamiento De Agua Potable En Colombia. I+D Revista de Investigaciones, 7 (1), 109-115.

\begin{abstract}
Resumen
Unos de los aspectos significativos en la selección de plantas convencionales de tratamiento de agua potable son los costos de inversión, dado que interrelacionan la capacidad de la planta, el cumplimiento normativo y la tecnología a aplicar según el tipo de agua cruda. Mediante el análisis de regresión exponencial, en esta investigación se utilizaron datos de 44 proyectos de plantas convencionales de tratamiento de agua potable nuevas, en donde se obtuvieron antecedentes de elasticidad de costo de escala en un crecimiento lento, en comparación con el caudal de diseño, para cada una de las plantas analizadas.
\end{abstract}

Palabras clave: Costos de inversión, agua potable, caudal.

\section{Abstract}

One of the significant aspects in the selection of conventional treatment plants for drinking water, are the investment costs, due to the relationship among the plant capacity, the compliance and the technology to be applied depending on the type of raw water. By means of an exponential regression analysis, in this paper we use data of 44 projects of conventional new treatment plants, where a history of elasticity cost of scale were obtained slow growth compared to the design flow for each of the plants analyzed were used.
Keywords: Investment costs, potable water flow.

\section{Introducción}

En la selección de la tecnología de la Planta de Tratamiento del Agua Potable (PTAP), se han tenido en cuenta factores como el grado de complejidad, el tipo de tecnología, el impacto directo en el área, la confiabilidad, la flexibilidad, el tiempo y el plazo, así como variables de calidad del agua cruda (turbiedad, color, $\mathrm{pH}$, conductividad, Coliformes fecales), el caudal y los costos directos del proyecto de PTAP (Carrión J. , 1992; Molinos M., 2010). Una PTAP es una productora de agua para consumo humano, según la normatividad del país que esté vigente. Hay que considerar así, la situación de adquisición de disposiciones técnico económicas, con base en un análisis de muchos casos empíricos, donde se trata de interrelacionar los costos de construcción, la tecnología a utilizar y el caudal de diseño de las PTAP.

En la literatura se encuentran aproximaciones al análisis económico de las PTAP, relacionando el costo de inversión o de construcción y el caudal de diseño, estableciendo de manera general una regresión en función de los costos y el caudal de las PTAP a nivel de estudios de factibilidad ( (Carrión J. , 1992) (Revollo D, 2010) (Valenzuela L.C, 2006) (Simate, 2015)), mediante una ecuación exponencial:

$$
C I=a Q^{b}
$$

'Artículo de investigación, con enfoque cuantitativo, resultado de un proyecto de investigación terminado, Perteneciente al área de Ingeniería Ambiental, Potabilización del agua, Desarrollado en el grupo de investigación: AQUAFORMAT, Financiado por la Universidad Distrital Francisco José de Caldas, Bogotá D.C., Colombia.

${ }^{2}$ Tecnóloga en Saneamiento Ambiental. Universidad Distrital Francisco José de Caldas. Facultad del Medio Ambiente y Recursos Naturales. Semillero de Investigación OBATALÁ. Bogotá-Colombia. Correo electrónico: karen_liliana0415@hotmail.com

${ }^{3}$ Tecnóloga en Saneamiento Ambiental. Universidad Distrital Francisco José de Caldas. Facultad del Medio Ambiente y Recursos Naturales. Semillero de Investigación OBATALÁ Bogotá-Colombia. Correo electrónico: ginalrey@hotmail.com

${ }^{4}$ Rodríguez Miranda Juan Pablo Profesor Asociado. Facultad del Medio Ambiente y Recursos Naturales. Universidad Distrital Francisco José de Caldas. Grupo de investigación: AQUAFORMAT. Correo electrónico: jprodriguezm@udistrital.edu.co 
Donde CI es el costo de inversión o de construcción, Q es el caudal de diseño de la PTAP, a y b son coeficientes calculados. Sin embargo, la constante "a" representa el costo de capacidad unitaria y la constante "b" se considera como la constante de elasticidad costo escala (siempre positiva). Si $b=1$ significa que los costos de inversión son directamente proporcionales a la capacidad o ca甘dal de la PTAP (los costos crecen de forma lineal). Si b 1, significa que los costos progresan menos que proporcionalmente a la capacidad o tamaño de la PTAP; es decir, se presenta una economía de escala que describe el comportamiento de los costos según la variable del caudal o tamaño (Revollo D, 2010). Para el parámetro "b" es menor en la función de costos de las PTAP, se considera que lentamente crece su costo a medida que se consíderan caudales o capacidades mayores de las PTAP. Si b 1, se presentaría una falsa economía de escala (Valenzuela L.C, 2006). Sin embargo, la literatura se reportan valores de la constante b entre 0.68 y 0.954 (Friedler E., 2006).

Lo anterior, ha servido para la planificación de nuevas PTAP, pero solo en el costo directo de construcción o de inversión aproximados a nivel de prefactibilidad para la asignación eficiente de recursos (Onkal G., 2006), medición de los impactos de los usuarios finales de un proyecto de PTAP, indica el orden de prioridad de la inversión, compara entre varios proyectos de PTAP, sin incluir aspectos como la localización, área, impactos ambientales y los precios locales en el momento de la construcción, entre algunos aspectos. Ciertas regresiones de exponenciales se han diferenciado según la tecnología convencional del tratamiento de agua potable, como lo muestra la Tabla 1:

Tabla 1.

Ecuaciones de costos de diversos países.

\begin{tabular}{|c|c|c|c|}
\hline TECNOLOGÍA & AÑO & FUENTE & ECUACION COSTO DE INVERSIÓN \\
\hline $\begin{array}{l}\text { FILTRACION RAPIDA } \\
\text { CONVENCIONAL }\end{array}$ & 1977 & CEPIS & $\begin{array}{c}C I=1656^{*} Q^{0.66} \\
\mathrm{Q}=\text { Caudal }\left(\mathrm{m}^{3} / \mathrm{s}\right) ; \mathrm{CI}=\text { Costo en miles dólares }\end{array}$ \\
\hline FILTRACION RAPIDA MODIFICADA & 1977 & CEPIS & $\begin{array}{c}C I=1194 * Q^{0.60} \\
\mathrm{Q}=\text { Caudal }\left(\mathrm{m}^{3} / \mathrm{s}\right) ; \mathrm{CI}=\text { Costo en miles dólares }\end{array}$ \\
\hline $\begin{array}{l}\text { FILTRACION ASCENDENTE } \\
\text { DESCENDENTE }\end{array}$ & 1977 & CEPIS & $\begin{array}{c}C I=1074 * Q^{0.59} \\
\mathrm{Q}=\text { Caudal }\left(\mathrm{m}^{3} / \mathrm{s}\right) ; \mathrm{CI}=\text { Costo en miles dólares }\end{array}$ \\
\hline FILTRACION ASCENDENTE & 1977 & CEPIS & $\begin{array}{c}C I=950 * Q^{0.59} \\
\mathrm{Q}=\text { Caudal }\left(\mathrm{m}^{3} / \mathrm{s}\right) ; \mathrm{CI}=\text { Costo en miles dólares }\end{array}$ \\
\hline SISTEMA CONVENCIONAL & 2010 & ACP (Panamá) & $\mathrm{CI}=1 \mathrm{USD} \$=1 \mathrm{Gal} / \mathrm{día}$ \\
\hline
\end{tabular}

fuente: Los autores

En Colombia, se han realizado varios análisis de

regresión de exponencial, yse han formulado funciones de costos para diversas tecnologías de PTAP, tal como se

Tabla 2.

Ecuaciones de costos para Colombia.

\begin{tabular}{|c|c|c|c|}
\hline TECNOLOGÍA & AÑO & FUENTE & ECUACION COSTO DE INVERSIÓN \\
\hline $\begin{array}{c}\text { SISTEMA } \\
\text { CONVENCIONAL }\end{array}$ & 1982 & INSFOPAL (Colombia) & $\begin{array}{c}C I=2078 * Q^{0.53} \quad(1 \leqslant \mathrm{Q} \leqslant 150 \mathrm{~L} / \mathrm{s}) \\
\mathrm{Q}=\text { Caudal }(\mathrm{L} / \mathrm{s}) ; \mathrm{CI}=\text { Costo en miles dólares }\end{array}$ \\
\hline $\begin{array}{c}\text { SISTEMA } \\
\text { CONVENCIONAL }\end{array}$ & 1982 & INSFOPAL (Colombia) & $\begin{array}{c}C I=437 * Q^{0.83} \quad(1 \leqslant \mathrm{Q} \leqslant 150 \mathrm{~L} / \mathrm{s}) \\
\mathrm{Q}=\text { Caudal }(\mathrm{L} / \mathrm{s}) ; \mathrm{CI}=\text { Costo en miles dólares }\end{array}$ \\
\hline $\begin{array}{c}\text { SISTEMA } \\
\text { CONVENCIONAL }\end{array}$ & 1998 & $\begin{array}{l}\text { Ministerio de Desarrollo } \\
\text { Económico (Colombia) }\end{array}$ & $\begin{array}{c}C I=70,52 * Q^{0.36}(2 \leqslant \mathrm{Q} \leqslant 100 \mathrm{~L} / \mathrm{s}) \\
\mathrm{Q}=\text { Caudal }(\mathrm{L} / \mathrm{s}) ; \mathrm{CI}=\text { Costo en miles COLPESOS }\end{array}$ \\
\hline $\begin{array}{l}\text { FILTRACION } \\
\text { DIRECTA }\end{array}$ & 1998 & $\begin{array}{l}\text { Ministerio de Desarrollo } \\
\text { Económico (Colombia) }\end{array}$ & $\begin{array}{c}C I=62.68 * Q^{0.32}(2 \leqslant \mathrm{Q} \leqslant 100 \mathrm{~L} / \mathrm{s}) \\
\mathrm{Q}=\text { Caudal }(\mathrm{L} / \mathrm{s}) ; \mathrm{CI}=\text { Costo en miles COLPESOS }\end{array}$ \\
\hline
\end{tabular}




\begin{tabular}{|c|c|c|c|}
\hline $\begin{array}{l}\text { FILTRACION } \\
\text { MULTIPLE ETAPAS }\end{array}$ & 1998 & $\begin{array}{l}\text { Ministerio de Desarrollo } \\
\text { Económico (Colombia) }\end{array}$ & $\begin{array}{c}C I=38,60 * Q^{0.67}(2 \leqslant \mathrm{Q} \leqslant 100 \mathrm{~L} / \mathrm{s}) \\
\mathrm{Q}=\text { Caudal }(\mathrm{L} / \mathrm{s}) ; \mathrm{CI}=\text { Costo en miles COLPESOS }\end{array}$ \\
\hline $\begin{array}{l}\text { FILTRACION } \\
\text { GRUESA Y DIRECTA }\end{array}$ & 1998 & $\begin{array}{l}\text { Ministerio de Desarrollo } \\
\text { Económico (Colombia) }\end{array}$ & $\begin{array}{c}C I=38,60 * Q^{0.67}(2 \leqslant \mathrm{Q} \leqslant 100 \mathrm{~L} / \mathrm{s}) \\
\mathrm{Q}=\text { Caudal }(\mathrm{L} / \mathrm{s}) ; \mathrm{CI}=\text { Costo en miles COLPESOS }\end{array}$ \\
\hline $\begin{array}{c}\text { SISTEMA } \\
\text { CONVENCIONAL }\end{array}$ & 2001 & $\begin{array}{l}\text { Alean, H.M.; Díaz - } \\
\text { Granados, M. (Colombia) }\end{array}$ & $\begin{array}{c}C I=13010^{*} Q^{0.8082} \\
\mathrm{Q}=\text { Caudal }(\mathrm{L} / \mathrm{s}) ; \mathrm{CI}=\text { Costo en miles COLPESOS }\end{array}$ \\
\hline $\begin{array}{l}\text { TEMA CONVENCIONAL } \\
\text { ompacta - Fibra de vidrio) }\end{array}$ & 2001 & $\begin{array}{l}\text { Alean, H.M.; Díaz - } \\
\text { Granados, M. (Colombia) }\end{array}$ & $\begin{array}{c}C I=21886^{*} Q^{0.5734} \\
\mathrm{Q}=\text { Caudal }(\mathrm{L} / \mathrm{s}) ; \mathrm{CI}=\text { Costo en miles COLPESOS }\end{array}$ \\
\hline $\begin{array}{c}\text { SISTEMA } \\
\text { CONVENCIONAL }\end{array}$ & 2005 & $\begin{array}{l}\text { Ministerio de Ambiente, } \\
\text { Vivienda y Desarrollo } \\
\text { Territorial (Colombia) }\end{array}$ & $\begin{aligned} C I & =91 * Q^{0.588} \\
\mathrm{Q}=\text { Caudal }(\mathrm{L} / \mathrm{s}) ; \mathrm{CI} & =\text { Costo en miles COLPESOS }\end{aligned}$ \\
\hline $\begin{array}{l}\text { TEMA CONVENCIONAL } \\
\text { ompacta - Fibra de vidrio) }\end{array}$ & 2006 & $\begin{array}{l}\text { Romero, A,; Rodríguez, } \\
\text { J.P. (Colombia) }\end{array}$ & $\begin{array}{c}C I=81231292.53 * Q^{2.837} \quad(0.5 \leqslant \mathrm{Q} \leqslant 1.5 \mathrm{~L} / \mathrm{s}) \\
\mathrm{Q}=\text { Caudal }(\mathrm{L} / \mathrm{s}) ; \mathrm{CI}=\text { Costo en COLPESOS } \\
C I=31467947.5 * Q^{2.536} \quad(1,5 \leqslant \mathrm{Q} \leqslant 3.0 \mathrm{~L} / \mathrm{s}) \\
\mathrm{Q}=\text { Caudal }(\mathrm{L} / \mathrm{s}) ; \mathrm{CI}=\text { Costo en COLPESOS } \\
C I=125155,7 * Q^{5.182} \quad(4.0 \leqslant \mathrm{Q} \leqslant 6.0 \mathrm{~L} / \mathrm{s}) \\
\mathrm{Q}=\text { Caudal }(\mathrm{L} / \mathrm{s}) ; \mathrm{CI}=\text { Costo en COLPESOS }\end{array}$ \\
\hline
\end{tabular}

Fuente: Los autores

muestra en la Tabla 2 .

\section{Tipo de investigación.}

\section{MÉTODO}

El tipo de investigación empleada en este desarrollo del modelo de función de costos de las PTAP puede ser establecido desde el ámbito prospectivo (Vergel G., 2010), dado que se registra información según va ocurriendo el fenómeno, en este caso, obtener los datos de costos de inversión futura de las PTAP en el Departamento de Cundinamarca; sin embargo, según el análisis y alcance de los resultados la investigación también puede ser cuasi experimental (Vergel G., 2010), debido a que existe una relación causal (causa - efecto), entre los costos de inversión, caudales de agua potable y parámetros físicos de la calidad del agua cruda, que en condiciones de control riguroso de los factores pueden afectar el resultado del análisis. Además, con las funciones de costos de las PTAP se podrá prever la asignación eficiente de recursos de inversión para la preservación y conservación de cuencas hídricas en el Departamento, por ello, la investigación también se denomina de pronóstico(Hurtado J., 2000), dado que se anticiparán situaciones futuras, en condiciones de horizonte de proyecto, demanda de agua potable, cumplimiento normativo del agua para consumo humano y el fortalecimiento institucional.

\section{Análisis de los datos}

Las funciones de costos en PTAP, que expresan una regresión exponencial de la forma $C I=\mathrm{a} Q$, donde $C I$ es el costo de inversión o de construcción, $\mathrm{Q}$ es el caudal de diseño, a y b son coeficientes calculados, son modelos que permiten establecer las relaciones entre variables e identificar la dependencia existente; es decir, se trata de un modelo determinista, pero que deja factores externos que producen fluctuaciones que influyen en la construcción dela función de costos, lo que significa que puede ser insuficiente para explicar la realidad del fenómeno. Por ello, la construcción de una función de costos de la PTAP, debe ser de forma estocástica o aleatoria, es decir, que incluya información externa desconocida (representa los factores que afectan la función de costos de las PTAP y que no son considerados en el modelo), mediante el error "", para que sea mejor explicado el modelo que interprete mejor la realidad(Gallego J., 2012).

\section{ANÁLISIS DE RESULTADOS Y DISCUSIONES.}

Para la construcción de la función de costos de inversión para las PTAP, se obtuvo información de 44 PTAP nuevas a construir en la fase de factibilidad, en tecnología convencional, semi-compacta y compacta, diferenciadas, y \$ạıuestran en las tablas 3, 4 y 5.

Plantas de tratamiento de agua potable tecnología convencional.

\begin{tabular}{cc}
$\begin{array}{c}\text { CAUDAL } \\
(\text { L/s) }\end{array}$ & $\begin{array}{c}\text { COSTO DIRECTO PTAP } \\
\text { CONVENCIONAL (USDS) }\end{array}$ \\
\hline 0.3 & 57769.2 \\
0.3 & 40943.1 \\
0.3 & 32954.1 \\
0.5 & 85160.3 \\
1.0 & 96005.8 \\
1.2 & 47310.8 \\
1.4 & 78235.5 \\
1.4 & 86533.0 \\
1.9 & 83352.3 \\
2.4 & 175198.4 \\
2.9 & 115219.1 \\
6.6 & 285106.3 \\
79.8 & 752013.8 \\
54.2 & 856551.1 \\
\hline
\end{tabular}

Fuente: Los autores 
Figura 1. Costo de inversión y caudal de diseño para PTAP Convencional

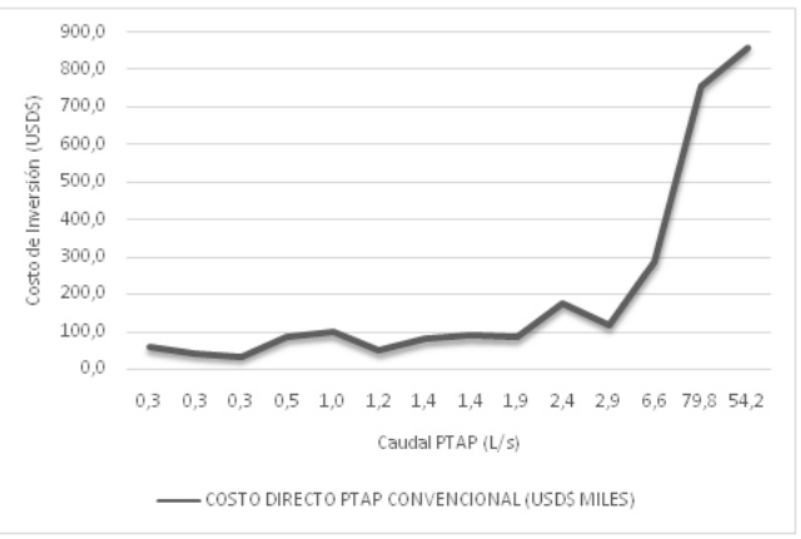

Fuente: Los autores

En la Tabla 3, se observan los 14 proyectos de PTAP con tecnología convencional según el caudal de diseño, en donde la tendencia es tener un caudal mayor y por ende un costo de inversión mayor. En la Figura 1, se observa el comportamiento de los costos de inversión según el caudal de diseño y costo de inversión para plantas de tratamiento de agua potable convencionales.

Tabla 4.

Plantas de tratamiento de agua potable tecnología

$\begin{array}{cc}\text { semicompastal } & \text { COSTO DIRECTO PTAP } \\ (\mathrm{L} / \mathrm{s}) & \text { SEMICOMPACTA (USDS) }\end{array}$

\begin{tabular}{lc}
0.3 & 8129.0 \\
0.5 & 9758.1 \\
1.0 & 9032.3 \\
1.5 & 10806.5 \\
2.0 & 12580.6 \\
3.0 & 15322.6 \\
4.0 & 18112.9 \\
5.0 & 22290.3 \\
6.0 & 29032.3 \\
7.0 & 33709.7 \\
8.0 & 38387.1 \\
10.0 & 40483.9 \\
\hline
\end{tabular}

Fuente: Los autores
Figura 2. Costo de inversión y caudal de diseño para PTAP

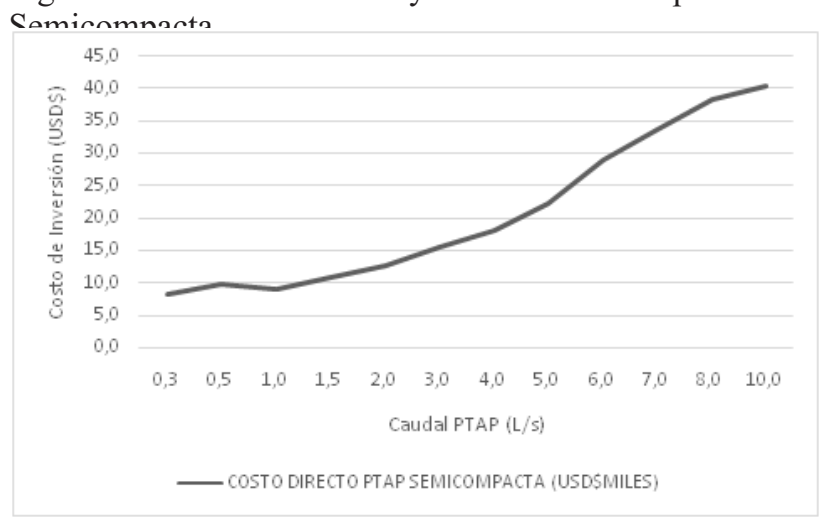

Fuente: Los autores

En la Tabla 4, se observan los 12 proyectos de PTAP con tecnología semicompacta según el caudal de diseño, en donde la tendencia es tener un caudal mayor y por ende un costo de inversión mayor. En la Figura 2, se observa el comportamiento de los costos de inversión según el caudal de diseño y costo de inversión, con una tendencia creciente uniforme.

Tabla 5.

Plantas de tratamiento de agua potable tecnología compacta CADAL COSTO DIRECTO PTAP (L/s) COMPACTA (USD\$)

\begin{tabular}{ll}
\hline 0.2 & 19682.6 \\
0.4 & 22489.0 \\
0.5 & 44603.9 \\
0.5 & 25900.7 \\
0.6 & 37765.0 \\
0.6 & 22301.9 \\
0.7 & 24172.9 \\
0.8 & 24172.9 \\
0.8 & 36353.4 \\
1.0 & 14790.7 \\
1.1 & 45612.2 \\
1.2 & 27166.5 \\
1.3 & 46957.7 \\
1.4 & 31513.1 \\
2.7 & 37681.3 \\
2.9 & 66431.1 \\
3.2 & 43710.3 \\
4.4 & 84384.5 \\
\hline
\end{tabular}

Fuente: Los autores 
Figura 3. Costo de inversión y caudal de diseño para PTAP

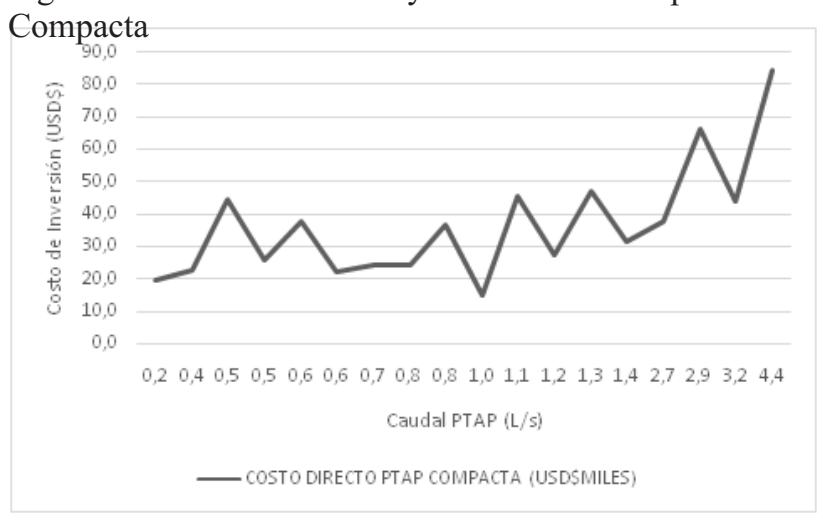

Fuente: Los autores

En la Tabla 5, se observan los 14 proyectos de PTAP con tecnología compacta según el caudal de diseño, en donde la tendencia es tener un caudal mayor y por ende un costo de inversión mayor. En la Figura 3, se observa el comportamiento variable de los costos de inversión según el caudal de diseño y costo de inversión de las plantas de tratamiento de agua potable, con una tendencia creciente uniforme.

\section{Escenario de análisis}

Este escenario considera que el modelo o función de costos es un log-log, donde se estima el logaritmo de inversión de la PTAP en dólares americanos contra el logaritmo de caudal o capacidad de la PTAP. Comparando estas dos ecuaciones, se puede apreciar que el coeficiente es un estimador de log (costo de inversión), mientras que el estimador de caudal es el exponente de la función exponencial.

Tabla 6.

\begin{tabular}{|c|c|}
\hline TECNOLOGIAA & $\begin{array}{l}\text { por modeto LOg-LOg. } \\
\text { ECUACION COSTO DE INVERSIÓN }\end{array}$ \\
\hline SISTEMA & $\mathrm{CI}=22038 * \mathrm{Q}^{0,9136}(5 \leqslant \mathrm{Q} \leqslant 80 \mathrm{~L} / \mathrm{s})$ \\
\hline CONVENCIONAL & $\mathrm{Q}=$ Caudal $(\mathrm{L} / \mathrm{s}) ; \mathrm{CI}=$ Costo en USD $\$$ \\
\hline SISTEMA & $\mathrm{CI}=18604 * \mathrm{Q}^{0,2851} \quad(0,1 \leqslant \mathrm{Q} \leqslant 5 \mathrm{~L} / \mathrm{s})$ \\
\hline $\begin{array}{c}\text { CONVENCIONAL } \\
\text { (Compacta - } \\
\text { Fibra de vidrio) }\end{array}$ & $\mathrm{Q}=$ Caudal $(\mathrm{L} / \mathrm{s}) ; \mathrm{CI}=$ Costo en USD $\$$ \\
\hline SISTEMA & $\mathrm{CI}=5477 * \mathrm{Q}^{0,7026} \quad(0,3 \leqslant \mathrm{Q} \leqslant 10 \mathrm{~L} / \mathrm{s})$ \\
\hline $\begin{array}{l}\text { CONVENCIONAL } \\
\text { (Semicompacta - } \\
\text { Fibra de Vidrio) }\end{array}$ & $\mathrm{Q}=$ Caudal $(\mathrm{L} / \mathrm{s}) ; \mathrm{CI}=$ Costo en USD $\$$ \\
\hline
\end{tabular}

\section{Fuente: Los autores}

En la Tabla 6, se observa que el modelo o la función de costos es la siguiente: la función de costos para las PTAP convencionales se expresa así: $C I=22038 * Q^{0.9136}$. El, valor del coeficiente expresa la elasticidad del caudal en función del costo que es de 0.9136 para el coeficiente de correlación de 0.5038 y rango de caudal de 5 a $80 \mathrm{~L} / \mathrm{s}$, lo que indica que si se incrementa el caudal o capacidad en un $1 \%$, el costo de la inversión de la planta de tratamiento de agua potable, aumentará en un $0.91 \%$ (menos de la unidad).

La función de costos para las PTAP compacta se expresa así: $C I=18604 * Q^{0.2851}$. El valor del coeficiente expresa la elasticidad del caudal en función del costo que es de 0.2851 para el coeficiente de correlación de 0.2677 y rango de caudal de 0.1 a $5 \mathrm{~L} / \mathrm{s}$, lo que indica que si se incrementa el caudal o capacidad en un $1 \%$, el costo de la inversión de la PTARM aumentará en un 0,28\% (menos de la unidad). Para la función de costos para las PTAP semicompacta se expresa así: $C I=5477 * Q^{0.7026}$. El valor del coeficiente expresa la elasticidad del caudal en función del costo que es de 0.7026 para el coeficiente de correlación de 0.8184 y rango de caudal de 0.3 a $10 \mathrm{~L} / \mathrm{s}$, lo que indica que si se incrementa el caudal o capacidad en un $1 \%$, el costo de la inversión de la PTARM aumentará en un $0.70 \%$ (menos de la unidad).

\section{CONCLUSIONES}

En términos de los costos de inversión para las PTAP, se evidencia una gran dispersión en los datos conseguidos, la dispersión de los datos y la fluctuación de los materiales, equipos, obras civiles al momento de la estimación del presupuesto, lo cual afecta sensiblementeel valor de cálculo(costo per cápita de inversión),comparado con la literatura internacional y latinoamericana; en gran medida asumiendo el costo de inversión de forma indirecta los habitantes de la población beneficiada, mediante la financiación a través del gobierno nacional, pero luego de forma directa mediante el costo de operación y mantenimiento de las plantas de tratamiento de agua potable.

Las funciones de costos de inversión de las PTAP formuladas, son una buena herramienta para la selección a nivel de prefactibilidad y, además, sirve como un indicador económico para la toma de decisión e inclusive para la evaluación entre varias alternativas de tratamiento del agua potable; en las ecuaciones de costos de inversión de PTAP (convencional, compacta y semicompacta), el coeficiente fue $\mathrm{b}<1$, lo cual significó que los costos progresan proporcionalmente menos que la capacidad de la PTAP, esto quiere decir, que se presenta una economía de escala, describiendo el comportamiento de los costos según la variable del caudal o tamaño, lo cual depende en gran medida de las características del agua cruda por tratar.

Ecuaciones Econométricas Para Los Costos De Inversión En Plantas De Tratamiento De Agua Potable En Colombia.

\section{AGRADECIMIENTOS}

Los autores agradecen a las Empresas Públicas de 
Cundinamarca S.A. E.S.P., dependencia dela Gobernación de Cundinamarca, Colombia, por la información suministrada de los proyectos de inversión de PTAP dentro del Plan Departamental de Aguas (PDA), en el marco del convenio con la Universidad Distrital Francisco José de Caldas.

\section{REFERENCIAS}

Carrión, J. (1992). El Agua - calidad y tratamiento para consumo humano. Lima, Perú: Centro Panamericano de Ingeniería Sanitaria y Ciencias Ambientales (CEPIS).

Carrión, J. (1992). Mejoramiento de calidad del aguapara consumo humano.LIMA: OPS/CEPIS.

Friedler E. (2006). Effects of design flow and treatment level on construction and operation costs of municipal wastewater treatment plants and their implications on policy making. Water research, 3751 - 3758.

Gallego J. (2012). Modelo económetrico básico. Teoría y conceptos. Alemania: Académica Española.

Hurtado J. (2000). Metodología de la investigación holística. . Caracas: Fundación SYPAL.

Molinos M. (2010). Economic feasibility study for wastewater treatment: a cost - benefit analysis. Science of the environmental, 4396 - 4402.

Onkal G. (2006). Cost analysis of alternative methods for wastewater handling in small communities. Journal of environmental management, 357 - 363 .
Revollo D. (2010). Análisis de las economías de escala y alcance en los servicios de acueducto y alcantarillado en Colombia. Desarrollo y sociedad, 145- 182.

Simate, G. (2015). The treatment of brewery wastewater for reuse by integration of coagulation/flocculation and sedimentation with carbon nanotubes sandwiched in a granular filter bed. Journal of Industrial and Engineering Chemistry, $1277-1285$.

Singhirunnusorn W. (2010). A critical analysis of economic factors for diverse wastewater treatment process: case studies in thailand. Sustain Environmental, 263 - 268.

Tsagarakis K.P. (2003). Application of cost criteria for selection of municipal wastewater treatment systems. Water, air and soil pollution, $187-210$.

Valenzuela L.C. (2006). Evaluación económica y metodología de minimización de costos para proyectos de sistemas de agua potable. Desarrollo y sociedad, 147- 171.

Vargas, L. (2006). Análisis de flujo y factores que determinan los periodos de retención. LIMA: OPS/CEPIS.

Vergel G. (2010). Metodología. Un manual para la elaboración de diseños y proyectos de investigación. Compilación y ampliación temática. Barranquilla: Publicaciones Corporación UNICOSTA. 